\title{
$\infty$
}

\section{Analysis on the Depth of Anesthesia by Using EEG and ECG Signals}

\author{
Soo-Young Ye, Seok-Yoon Choi, and Dong-Hyun Kim ${ }^{+}$ \\ Department of Radiological Science, Catholic University of Pusan, Busan 609-757, Korea \\ Seong-Hwan Song \\ Division of Energy and Bio-engineering, Dongseo University, Busan 617-716, Korea
}

Received July 18, 2013; Revised August 14, 2013; Accepted September 2, 2013

\begin{abstract}
Anesthesia, which started being used to remove pain during surgery, has become itself one of the major concerns to be considered during surgery. While actual anesthesia is being performed, patients tend to have unpleasant experiences, due to wakening that accompanies pain, or wakening that does not accompany pain. Since this awakening during anesthesia is a most unpleasant experience in a patient's life, evaluating the depth of anesthesia during surgery is essential for patients to avoid this experience. Although there has been much effort on the understanding and measurement of the depth of anesthesia, while various researches were performed on the need of anesthesia, the development of an indicator that could objectively evaluate the depth of anesthesia, other than by using the patient's vital signs, is still inadequate. Therefore, this study was to develop an objective indicator by using EEG and ECG, which are essentially measured during the surgery, to evaluate the depth of anesthesia. The experiment was performed by taking patients who require a relatively short operation time, and general inhalation anesthetics among surgical patients in obstetrics and gynecology as the subjects of experiment, to measure the EEG and ECG signals of patients under anesthetics. The result showed that SEF using EEG and LF, HF using ECG signal and correlation dimension analysis parameter were valuable parameters that could measure the depth of anesthesia, by the stage of anesthesia.
\end{abstract}

Keywords: Depth of anesthesia, EEG, ECG, PSD, LF, HF

\section{INTRODUCTION}

Anesthesia means the dissipation of consciousness, sensory blockade, motor blockade, or reflection blockade, and it includes not only the act that injects the anesthetic, but also all the actions to safely maintain the patient's condition from before the surgery, until the patient fully wakes up from anesthesia in the recovery room, after surgery. Therefore, a clinician in the department of anesthesiology should understand the disease and whole body condition of the patient before the surgery, and

${ }^{\dagger}$ Author to whom all correspondence should be addressed: E-mail:dhkim@cup.ac.kr

Copyright $@ 2013$ KIEEME. All rights reserved. This is an open-access article distributed under the terms of the Creative Commons Attribution Non-Commercial
License (http:///creativecommons.org/licenses/by-nc/3.0) which permits unrestricted noncommercial use, distribution, and reproduction in any medium, provided the original work is properly cited performs a wide range of treatment, from choosing an apposite anesthetizing method and medicine according to the kind of surgery and condition of the patient, to managing the condition of the patient after surgery.

The major elements of anesthesia consist of unconsciousness, amnesia, analgesia, proper muscle relaxation, and harmful loss of control of reflexes of the autonomic nervous system. In other words, it could be called a proper anesthesia only when it could provide an easy preparative state for surgery with no memory or consciousness, it maintains a stable condition of the vital signs, the reflection to external stimulus slows down, and a sufficient amount of muscle relaxation is achieved. To achieve this, not only the hemodynamic observation, such as blood pressure or pulse and observation on muscle relaxation, but also objective evaluation of the depth of anesthesia is vital. Also, as a serious psychological wound, awakening happening during the 
anesthesia can be causative of sleep disorder after the surgery, nightmare, and reminiscence, in addition to anguish from pain or helplessness feeling and sensation of fear during the surgery; thus proper monitoring on the depth of anesthesia is needed to prevent this.

Although there was much effort on the understanding and measurement of the depth of anesthesia, as various researches were carried out on the need of anesthesia, the development of an index that could objectively evaluate the depth of anesthesia, other than using the vital signs of the patient, is still inadequate.

Recently, researches have been carried out on the effect on the ANS (Autonomic Nervous System) using EEG (electroencephalogram) [1], esophageal contraction, AEP (auditory evoked potential) [2], SEF (spectral edge frequency) [3] and HRV (Heart rate variability) analysis of ECG (electrocardiogram) signal [4].

Research results that indicate when to maintain anesthesia, by judging the awareness condition with monitoring during the surgery using the EEG and ECG signals, minimize excessive or insufficient use of anesthetic; hence, it is easy to control anesthetic during the surgery, and also results showing fast recovery have been reported [5]. It is known that the BIS (bispectral index) number using the EEG signal as an index that shows the depth of anesthesia, reflects the depth of anesthesia by inhalation anesthetics or intravenous anesthetics [6]. Also, research that has addressed the spectrum of HRV during surgery has analyzed the LF (low-frequency), which is influenced by both the sympathetic nervous system and parasympathetic nervous system, and the HF (high-frequency) which is mainly influenced by respiration related to the parasympathetic nervous system [7], and observed the effect of ANS during anesthesia, by studying the change of HRV after spinal anesthesia [8]. Also, research on the activity of parasympathetic has progressed by analyzing what kind of correlation HRV has in general anesthesia with the Poincare plot analysis method [9], and research about the handling of noise for HRV analysis is also included as an important part of the research [10]. Although up to now, the depth of anesthesia during surgery is measured by using the EEG signal and ECG signal, these only provide an auxiliary role to the judgment of the clinician, but could not provide an objective standard of judgment.

This study tried to develop a subjective index that can measure the depth of anesthesia more accurately, by using both the EEG signal that reflects the effect of the central nervous system, and the ECG signal that reflects the effect of ANS.

\section{EXPERIMENTAL METHODS}

\subsection{Materials and method}

Patients of the ASA (American Society of Anesthesiologists) who were either I (healthy patient) or II (light systemic disease, no limit of function) that the society of anesthesiology units provided were taken as subjects. They were excluded when they had a medical history of cardiac disorder, high blood pressure, ANS, or when they were taking a blood pressure control drug, since it affects the acquired signal.

Patients who have a relatively short operation time and taking inhalation anesthesia procedure among surgical patients in maternity units were taken as experiment objects to measure the EEG and ECG signals of patients under anesthesia, and the EEG data was acquired after the agreement of patients, by explaining the purpose of the study to the subject patients before the surgery.

The average age of subject patients was $47.7 \pm 9.1$, and average weight was $70.7 \pm 10.5 \mathrm{~kg}$. As a pre-surgical step, pre-anesthetic medication glycopyrrolate $0.004 \mathrm{mg} / \mathrm{kg}$ and midazolam 0.05 $\mathrm{mg} / \mathrm{kg}$ were intramuscularly injected in the ward 30 minutes before the surgery. The experiment was carried out by dividing the surgical steps into pre-op, induction, operation, awakening and post-op. The pre-op step was acquired in the ward one day before the surgery, the induction was classified until the endotracheal intubation was operated, the operation step was classified from the end of the induction step to the beginning of anesthesia recovery, and the awakening step was classified from the end of the injection of anesthetic medicine. Although data of the post-op step should be acquired in the recovery room, it proved inconvenient to measure there, due to the characteristics of the recovery room, so it was measured by the patient waiting in the ward, who was fully recovered one day after the surgery.

A bio-signal measuring instrument (PhysioLab 400, PhysioLab Co., Korea) was used to acquire the EEG and ECG signal. An adhesive patch type of disposable Ag-AgCl electrode was used for the measurement of EEG, and the EEG was measured by using frontal lobe FP1 electrode displacement from the ten-twenty electrode system of the International Federation of the Societies for Electroencephalography and Clinical Neurophysiology for the attachment location of the electrode.

For measurement of the ECG signal, the lead I signal was acquired from the sampling ratio, by attaching the cathode and earth electrode on the left arm, and the negative pole on the right arm.

\subsection{Methods of signals analysis}

\subsubsection{Analysis of EEG signal}

Preprocessing, base line correction, and linear detrend technique was applied, to facilitate analysis of the EEG data. SEF was found by using a spectrum analytic technique from the EEG data that went through preprocessing.

FFT (Fast Fourier Transform) was performed, to convert time series EEG data to the frequency domain. The parameter that evaluates the depth of anesthesia that is being applied in clinic is $95 \% \mathrm{SEF}$, and the formula to extract these parameters can be presented as shown below.

$$
95 \% \text { SET }>\frac{T P}{100} \times 95
$$

Here, TP (total power) describes the size of the whole PSD (power spectrum density) of the EEG data, and 95\% SEF means the frequency of the spot where it reaches $95 \%$ of the PSD.

\subsubsection{Analysis of ECG signal}

The RR interval time series signal was found by detecting the $\mathrm{R}$ peak from the acquired ECG signal, and the time series HRV signal that is used in research was reformed, by preprocessing this signal. The influence of ANS was evaluated by applying the HRV signal with the method of frequency analysis and chaos analysis.

\section{(1) Analysis of frequency}

This research tried to evaluate the depth of anesthesia, by finding the PSD in each frequency domain by FFT 10 minutes of HRV signal in the anesthesia phase type. To achieve this, PSD was found, by classifying it into three frequency domains: HF, LF and VLF. Each frequency domain has a component for each major frequency domain that is related to various physiological mechanisms [11]. The influence of ANS by $1 / \mathrm{f}$ noise distribution characteristic was evaluated, as well as the PSD distribution 
characteristic for each frequency domain. $1 / \mathrm{f}$ noise means the power spectrum $\mathrm{P}(\mathrm{f})$ is a function of frequency $\mathrm{f}$; in other words, noise that is shown in the form of $P(f)=1 / f^{\beta}$. Here, since $\beta$ actually appears almost close to 1 , call it $1 /$ f. $\beta$ means the grade that appeared as the first linear regression in the graph of $\log P S D$ to $\log$ $\mathrm{f}[12]$.

\section{(2) Analysis of chaos}

A signal generated from a random power dynamic system has the information of that dynamic system. Therefore, the process that properly restructures the acquired time series data on the phase space to know the condition of that system is called embedding, and the coordinate of time delay was used for the method to restructure on the phase space [13]. In this research, the delay time was found with the AMI (average mutual information) method that could exclude the influence of noise, by using joint distribution probability among the data that comes from nonlinearity, with low autocorrelation of the bio-signal that occurs nonlinearly or semi-regularly [14]. In order to understand the dynamic characteristics of the measured value from any system, an attractor that reflects that dynamic information should be composed, and there is a need to accurately find embedding dimension that includes the attractor. The embedding dimension was decided by using a FNN (false nearest neighbor) algorithm [15], which is relatively insensitive to the size of data and noise in this research. The time series data produced from a chaos dynamic system has a geometrically self-similarity structure by stretching and folding, thus it has the fractal dimension, but a integral dimension. The condition of a system can be regulated by using the fractal dimension of non-integer that quantified self similarity, which is one of the characteristics of a chaos dynamic system [16]. The Correlation Dimension is a fractal dimension of attractor on the phase space, and it is an amount that quantifies the global geometry and local distribution of the attractor. Generally, the dimension of phase space is related to the number of differential equations of that system; and on the other hand, the dimension of an attractor is defined as the domain of phase space that is occupied by data points or the trace of the data points. Therefore the value of the correlation dimension is greater, the system has a more complex structure $[17,18]$.

\section{RESULTS}

In order to objectively analyze the influence of the central nervous system and autonomic nervous system for each anesthesia phase during the anesthesia, focusing on distinctive events written on the anesthesia record, the phase of anesthesia is divided into 5 phases. These 5 phases are pre-anesthesia, induction, operation, awakening and post-op. All of the subject patients used the anesthesia medicine enflurance, and the result of experiment was taken from the patients who had 1 hour 30 minutes to 2 hours 30 minutes of operation time, by applying EEG signal and ECG signal analysis.

\subsection{Analysis of EEG signal}

Figure 1 shows the SEF analysis result of the EEG signal in each anesthesia phase.

As is shown in Fig. 1, the tendency could be observed that the phase changes: 30.73 in pre-op, 26.91 in induction, 24.62 in operation, 29.59 in awakening, and 30.54 in post-op. The awakening step that is before and after the operation, showed 30 or more of value, and the phase of induction, operation and awakening, which is influenced by anesthesia medicine, showed 30 or less of

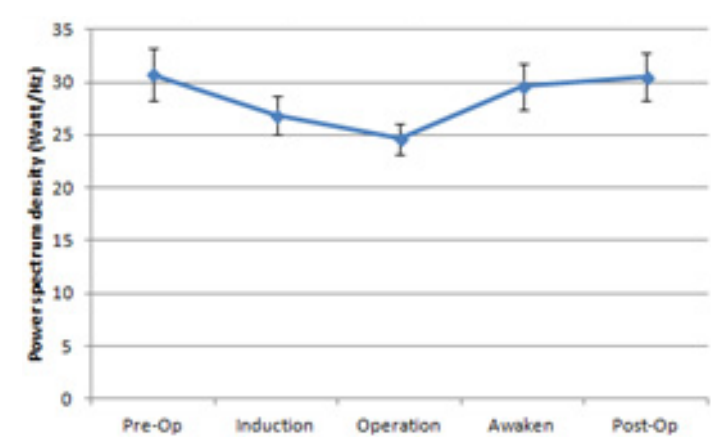

Fig. 1. The results of SEF analysis in each anesthesia stages.

value. The induction and awakening phase reflect the disappearance of consciousness by the injection of anesthesia medicine, and consciousness recovery by the blocking of anesthesia medicine, thus it showed the median of the condition in the surgical operation and awakening before and after the operation. The operation step that is in complete anesthesia showed the lowest average value of 24.62. From this result, it is observed that the SEF parameter relatively well reflects the phase of anesthesia as the anesthesia proceeds.

\subsection{Analysis of ECG signal}

Figure 2 shows the analysis result of LF, HF, LF/HF and 1/f noise, which is the parameter of the frequency domain. The LF parameter shows a very high PSD value in the pre-anesthesia, and post-anesthesia steps, and very low PSD in the operation step. There was great individual standard deviation value in the pre-anesthesia and post-anesthesia steps. However, the preanesthesia step and post anesthesia step were clearly classified, without overlapping with the next step induction, and the previous step awakening. Also, it showed a similar statistic result from $\mathrm{p}$ verification in the induction, operation, and awakening step.

The HF parameter showed a very high PSD value in preoperation and post-operation, and similarly was found from $\mathrm{p}$ verification in the induction, operation and awakening steps.

It showed a similar aspect in the LF/HF parameter and grade parameter of $1 / \mathrm{f}$ nose; however, the standard deviation was too big, and did not show a significant difference in each step of anesthesia, thus there was no possibility for this to be used as a parameter that could classify the depth of anesthesia in each anesthesia step.

As shown in Fig. 3, the correlation dimension parameter had a very high value of $4.3 \pm 0.6$ and $4.1 \pm 0.4$ in pre and postoperation. However, it rapidly reduced to $1.7 \pm 0.3$ in induction, and it reduced again to $1.3 \pm 0.5$ in the operation step. However, it increased again in the awakening step to $3.3 \pm 0.5$, and rapidly increased to $4.1 \pm 0.4$ in post-operation; thus it approached the preoperation step. Since the correlation dimension parameter has a low standard deviation for each patient, there is similarity in the $p$ verification, thus it is confirmed that it is a very useful parameter to distinguish the phase of anesthesia.

\section{CONCLUSIONS}

The SEF value using EEG of 30 or more of value in the awakening step, which is before and after the surgery, could be used to classify the anesthesia step. It is observed that the SEF parameter relatively well reflects the anesthetic step by the progress of anesthesia. Among the frequency domain analysis method using ECG 
(a)

\section{LF}

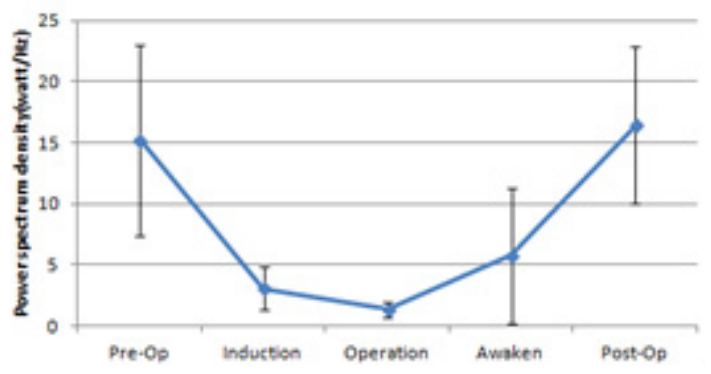

(b)

HF

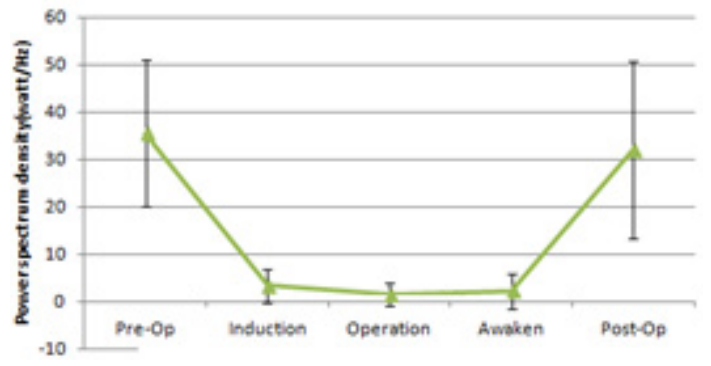

(c)

LF/HF

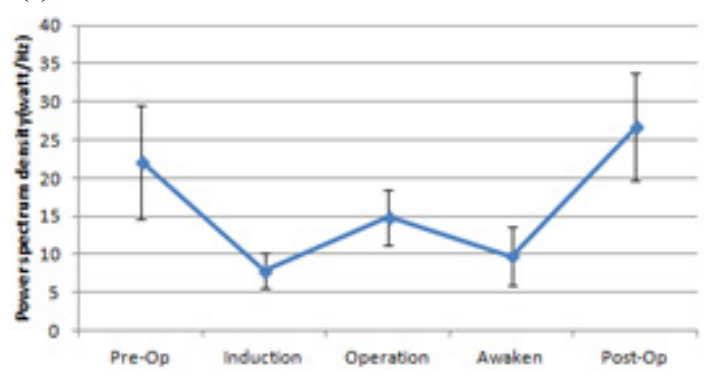

(d)

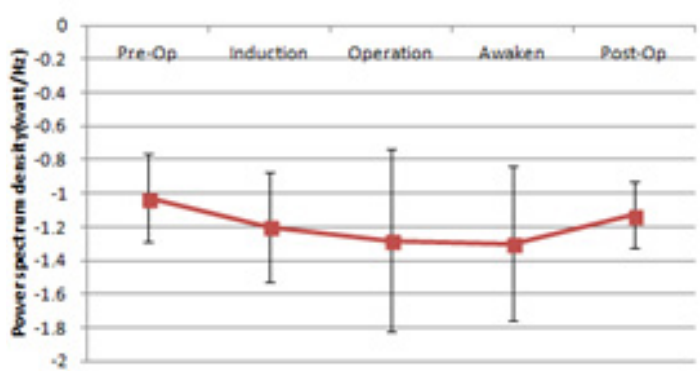

Fig. 2. The result of frequency of ECG signal (a) LH analysis, (b) HF analysis, (c) LF/HF analysis, and (d) $1 / \mathrm{f}$ noise analysis.

signal, the LF and HF value can distinguish when the subject is under anesthesia, and when he or she is not. This indicates the activity of ANS significantly decreased with anesthesia. It shows a high LF value with $15.2 \pm 7.8$ in pre-operation, $16.4 \pm 6.4$ in postoperation, and it is thought that this is because of the big influence of ANS, rather than the operation, in before and after the anesthesia. It rapidly decreased in the induction step, decreased again in the operation step, but increased again to $5.8 \pm 5.5$ in the awakening step. It is thought that this phenomenon is because although it is permitted to load in the respiration meter during the operation, the load is removed from the respiration meter in the awakening step, and hence the activity of the respiratory center of the ANS and pressure receptor of the sympathetic nervous system. It was rapidly increased to $16.4 \pm 6.4$ in the postanesthesia step.
CD

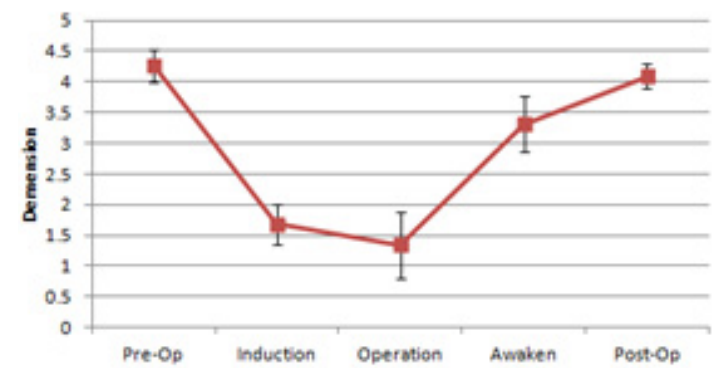

Fig. 3. The result of chaos analysis of ECG signal (correlation dimension).

The result of HF was similar to the result of LF. The overall analysis result of LF and HF is as follows. Since the distribution characteristic of PSD in the pre and post-anesthesia step is wide, and the average \pm standard deviation is very high, it is significantly decreased in the induction step, compared to the preoperation step.

Since the value of ratio LF/HF, which is well known to reflect the balance of ANS, and the $1 / \mathrm{f}$ noise showed too high a standard deviation for each patient in the aspect during the anesthesia, it was difficult to distinguish the depth of anesthesia. Maintaining the HRV signal reformed by using the ECG signal in $4.3 \pm 0.6$ from the correlation dimension in chaos analysis, it significantly dropped to $1.7 \pm 0.3$, compared to the induction step, compared to pre-anesthesia. This indicates the human body is definitely in a different condition, due to the anesthetic medicine injected during the induction step. Among the anesthesia without external stimulus, the value of correlation dimension was maintained at $1.3 \pm 0.5$. When inhalation anesthetic is ceased for the closure of the operation, the human body slowly wakes up from the anesthesia. The value of the correlation dimension in this step is very high, at $3.3 \pm 0.5$. This phenomenon is because the patient feels the pain from the laryngoscope in the organization, as he or she wakes up from the anesthesia. It is observed that the correlation dimension value increased again to the pre-operation condition in the post-operation step, with $4.1 \pm 0.4$.

As per the synthetically analyzed experiments above, it is confirmed that SEF using EEG, and the LF, HF, correlation dimension analysis parameter using the ECG signal is a valuable parameter that could evaluate the depth of anesthesia in each anesthetic step.

\section{ACKNOWLEDGMENT}

This paper was supported by RESEARCH FUND offered from Catholic University of Pusan.

\section{REFERENCES}

[1] John ER, "A field theory of consciousness", Conscious congnition, Vol. 10, No. 2, pp. 184 213, 2001 [DOI: http://dx.doi. org/10.1006/ccog.2001.0508].

[2] Recart A, White PF, Wang A, Gasanova I, Byerly S, Jones SB., "Effect of auditory evoked potential index monitoring on anesthetic drug requirements and recovery profile after laparoscopic surgery: a clinical utility study", Anesthesilolgy, Vol. 99, No. 4, pp. 813 818, 2003 [DOI: http://dx.doi.org/10.1213/ ane.0b013e3181adc21a].

[3] McKeever S, Johnston L, Davidson AJ.," An observational study 
exploring amplitude-integrated electroencephalogram and spectral edge frequency during paediatric anaesthesia." Anaesth Intensive Care, Vol. 40, No. 2, pp. 275 284, 2012 [DOI: http://dx.doi.org/10.1093/bja/aes312].

[4] Janda M, Schubert A, Bajorat J, Hofmockel R, Nöldge-Schomburg GF, Lampe BP, Simanski O., "Design and implementation of a control system reflecting the level of analgesia during general anesthesia”, Biomed Tech(Berl), Vol. 58, No. 1, pp. 1 11, 2013 [DOI: http://dx.doi.org/10.1515/bmt-2012-0090].

[5] L. Gugino, R. Chabot, L. Prichep, E. John, V. Formanek, and L. Aglio, "Quantitative EEG changes associated with loss and return of consciousness in healthy adult volunteers anaesthetized with propofol or sevoflurane," Br. J. Anaesth., Vol. 87, pp. 421 428, Sep. 2001 [DOI: http://dx.doi.org/10.1093/ bja/87.3.421].

[6] A. Miller, J.W.Sleigh, J.Barnard, and D.A.Steyn-Ross, "Does bispectral analysis and anything but complexity? BIS sub-components may be superior to BIS for detection of awareness ", $\mathrm{Br}$ J Anaesth., Vol. 93, No. 4, pp. 596 597, 2004 [DOI: http://dx.doi. org/10.1093/bja/aeh612].

[7] Curtis BM, O’Keefe JH,” Autonomic Tone as a Cardiovascular RiskFactor", The Dangers of Chronic Fight and Flight Mayo Clinic Proceedings, Vol. 77, No. 1, pp. 45 54, 2002 [DOI: http:// dx.doi.org/10.1016/S0025-6196(11)62137-X].

[8] A. T. Mazzeo, E. La Monaca, R. Di Leo, G. Vita and L. B. Santamaria, "Heart rate variability: a diagnostic and prognostic tool in anesthesia and intensive care " Acta Anesthesiol Scand, Vol 55, pp. 79 811, 2011 [DOI: http://dx.doi.org/10.1111/j.13996576.2011.02466.x].

[9] C. K Karmakar, A. H Khandoker, A. Voss and M. Palaniswami, "Sensitivity of temporal heart rate variability in Poincaré plot to changes in parasympathetic nervous system activity", BioMedical Engineering, Vol. 10:17, 2011 [DOI: http://dx.doi. org/10.1186/1475-925X-10-17].

[10] U. Kraus, A. Schneider, S. Breitner, R. Hampe, R. Rücker, M. Pitz, U. Geruschkat, P. Belcredi, K. Radon, A. Peters,” Individual Daytime Noise Exposure during Routine Activities and Heart Rate Variability in Adults: A Repeated Measures Study”, Environment Health perspectives, Vol. 121, pp. 607 612, 2013 [DOI: http://dx.doi.org/10.1289/ehp.1205606].

[11] Suzuki N, Sugawara J, Kimura Y, Nagase S, Okamura K, Yaegashi N.," Assessment of maternal heart-rate variability during labor using wavelet-based power spectral analysis", Gynecol Obstet Invest., Vol. 74, No. 1, pp. 35 40, 2012 [DOI: http://dx.doi. org/10.1159/000336064].

[12] Freeman WJ, Zhai J.," Simulated power spectral density (PSD) of background electrocorticogram (ECoG).", Cogn Neurodyn., Vol. 3, No. 1, 2009 [DOI: http://dx.doi.org/10.1007/s11571-0089064-y].

[13] Liao F, Jan YK., "Using recurrence network approach to quantify nonlinear dynamics of skin blood flow in response to loading pressure.", Conf Proc IEEE Eng Med Biol Soc., Vol. 2012, pp. 4196-9, 2012 [DOI: http://dx.doi.org/10.1109/ EMBC.2012.6346892].

[14] Benitez R, Alvarez-Lacalle E, Echebarria B, Gomis P, Vallverdu M, Caminal P.," Characterization of the nonlinear content of the heart rate dynamics during myocardial ischemia",Med Eng Phys. Vol. 31, No. 6, pp. 660-7, 2009 [DOI: http://dx.doi. org/10.1016/j.medengphy].

[15] Isgum I, Prokop M, Niemeijer M, Viergever MA, van Ginneken B., "Automatic coronary calcium scoring in low-dose chest computed tomography."IEEE Trans Med Imaging. Vol. 31, No.12, pp. 2322-2334, 2012 [DOI: http://dx.doi.org/10.1109/ TMI.2012.2216889].

[16] Lewis MJ, McNarry MA.,"Influence of age and aerobic fitness on the multifractal characteristics of electrocardiographic RR time-series.", Front Physiol., Vol. 4, No. 100, pp. 1 13, 2013 [DOI: http://dx.doi.org/10.3389/fphys].

[17] Xiuqing Zheng, Zhiwu Liao, Shaoxiang Hu, Ming Li, Jiliu Zhou, "Improving Spatial Adaptivity of Nonlocal Means in Low-Dosed CT Imaging Using Pointwise Fractal Dimension”, Comput Math Methods Med., Vol. 2013, No. 902143, 2013 [DOI: http://dx.doi. org/10.1155/2013/902143].

[18] Peter Grassberger, Itamar Procaccia. "Measuring the Strangeness of Strange Attractors". Physica D: Nonlinear Phenomena, Vol. 9 No. 1 2, pp. 189 208, 1983 [DOI: http://dx.doi. org/10.1016/0167-2789(83)90298-1] . 